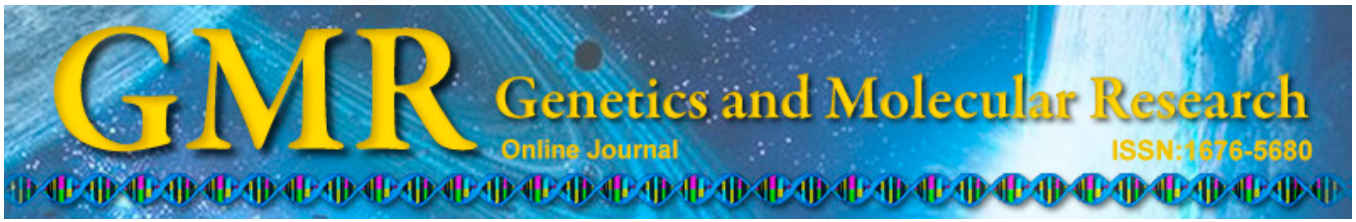

\title{
Gene expression profiling analysis of 5-hydroxytryptamine signaling pathway in rat regenerating liver and different types of liver cells
}

\author{
C.F. Chang ${ }^{1,2}$, J. Yang ${ }^{1,2}$, W.M. Zhao ${ }^{1,2}$, Y. Li ${ }^{1,2}$, P.J. Guo ${ }^{1,2}$, M.H. Li ${ }^{1,2}$, \\ Y. Zhou ${ }^{1,2,3}$ and C.S. Xu ${ }^{1,2}$
}

${ }^{1}$ Key Laboratory for Cell Differentiation Regulation, Xinxiang, Henan, China ${ }^{2}$ College of Life Science, Henan Normal University, Xinxiang, Henan, China ${ }^{3}$ College of Computer and Information Engineering, Henan Normal University, Xinxiang, Henan, China

Corresponding author: C.S. Xu

E-mail: cellkeylab@126.com

Genet. Mol. Res. 14 (2): 3409-3420 (2015)

Received May 30, 2014

Accepted August 27, 2014

Published April 15, 2015

DOI http://dx.doi.org/10.4238/2015.April.15.4

ABSTRACT. We examined the gene expression profiles of the
5-hydroxytryptamine signaling pathway in the regenerating liver and 8
types of liver cells during rat liver regeneration, and explored expression
differences in 5-hydroxytryptamine signaling pathway genes at the
level of tissues and cells, as well as the role of the pathway on liver
regeneration. Eight types of rat regenerating liver cells were isolated
using Percoll density-gradient centrifugation and immunomagnetic
bead methods. Rat Genome 2302.0 Array was used to detect
expression changes in 5-hydroxytryptamine signaling pathway genes.
The results showed that 26, $47,8,21,16,19,22,27$, and 20 genes
changed significantly in hepatocytes, biliary epithelial cells, hepatic
stellate cells, oval cells, sinusoidal endothelial cells, Kupffer cells, pit
cells, dendritic cells, and the regenerating liver, respectively. Synthetic 
effects of 5-hydroxytryptamine signaling pathway genes in 8 types of liver cells showed that 26 genes were expressed significantly; the expression trends of 10 genes were the same in the regenerating liver, while others were different. Based on the gene expression profiles of the 8 types of liver cells, 5-hydroxytryptamine promoted hepatocyte proliferation through the RAS and STAT3 signaling pathways, proliferation and differentiation of sinusoidal endothelial cells through the STAT3 signaling pathway, and proliferation and apoptosis of pit cells through the AKT3 signaling pathway. There were large differences in genes involved in 5-hydroxytryptamine signaling at the tissue and cellular levels; thus, liver regeneration should be studied indepth at the cellular level to reveal the molecular mechanism of liver regeneration.

Key words: 5-Hydroxytryptamine; Gene expression profiling analysis; Rat Genome 2302.0 microarray; Rat liver regeneration

\section{INTRODUCTION}

Liver regeneration (LR) is a very complex biological process (Lesurtel et al., 2006). After partial hepatectomy $(\mathrm{PH})$, a large number of genes are involved in liver regeneration, including cytokines, growth factors, and metabolism genes, which form 3 class networks (Fausto et al., 2006). Studies have shown that 5-hydroxytryptamine (5-HT) is a growth factor that plays a role in liver regeneration. After liver injury, the expression levels of the 5-HT receptors HTR2A and HTR2B increase in cells, stimulating cell growth and repairing the damaged liver after receptor binding in the blood (Fausto et al., 2006). However, the rat liver consists of many cell types, including hepatocytes (HCs), biliary epithelial cells (BECs), oval cells (OCs), hepatic stellate cells (HSCs), sinusoidal endothelial cells (SECs), Kupffer cells (KCs), pit cells (PCs), and dendritic cells (DCs), among others (Michalopoulos, 2007). Therefore, examining the effect of 5-HT on cells and tissues is important for revealing the molecular mechanism of LR at the cellular and transcriptional levels (Taub, 2004). Thus, we isolated 8 liver cell types using a combination of the Percoll density-gradient centrifugation and immunomagnetic bead methods, and the Rat Genome 2302.0 Array was used to determine the gene expression profiles of 8 liver cell types and the regenerating liver (RL) during rat LR. The relevance of 5-HT signaling pathway genes and LR was analyzed using bioinformatic and system biology methods. Our results provide a basis to further examine the regulation of 5-HT signaling pathway genes on liver regeneration.

\section{MATERIAL AND METHODS}

\section{$2 / 3$ hepatectomy in rats and liver regeneration}

A total of 114 Sprague-Dawley rats, weighting $230 \pm 20$ g provided by the Animal Center of Henan Normal University, were randomly divided into 9 (PH) groups, 9 operation control groups, and 1 normal control group, with 6 rats in each group (male:female $=1: 1$ ). Rats in the PH groups underwent operation to remove $70 \%$ of their livers as previously de- 
scribed by Higgins and Anderson (1931). Briefly, the left and median lateral liver lobes were surgically removed, and the rats after hepatectomy were normally fed for $0,2,6,12,24,30$, $36,72,120$, and $168 \mathrm{~h}$. The operation control group was subjected to the same procedure as the $\mathrm{PH}$ group but without liver removal.

\section{Isolation and identification of 8 liver cell types}

Rats were subjected to abdominal skin disinfection with alcohol after anesthetization by diethyl ether inhalation. The abdominal cavity was opened to expose the liver, and the portal vein was catheterized, the inferior vena cava tied, and the thoracic inferior vena cava severed. Next, a conventional 2-step method was applied for scattering the liver cell suspension (Kreamer et al., 1986). At termination, the suspension was filtered through nylon mesh (200mesh) to separate the mixed cell populations. Only the mixed cell populations with a separation efficiency of $\geq 5.0 \times 10^{8}$ cells/rat, cell viability $\geq 95 \%$, and red cells $\leq 0.1 \%$ were used. The samples collected from 4 rats per group were mixed and adjusted to $1 \times 10^{8}$ cells $/ \mathrm{mL}$ (Haber et al., 1993; Alpini et al., 1994; Jurima-Romet et al., 1991, 1996). Next, $6 \mathrm{~mL}$ mixed cell suspension was spread onto the surface of $4 \mathrm{~mL} 60 \%$ Percoll in a $10-\mathrm{mL}$ tube for centrifugation at $200 \mathrm{~g}$ for $5 \mathrm{~min}$ at $4^{\circ} \mathrm{C}$. The centrifuged pellets and supernatant contained purified hepatocytes (Vondran et al., 2008) and nonparenchymal cell-enriched supernatant fractions (Duret et al., 2007), respectively. The supernatant was then centrifuged at $400 \mathrm{~g}$ for $2 \times 2 \mathrm{~min}$ at $4^{\circ} \mathrm{C}$, and the harvested pellet contained nonparenchymal cells. The nonparenchymal cell populations were incubated with $10 \mu \mathrm{L} / \mathrm{mL}$ rat anti-PE-GFAP, -CD68, -CD31, -CK19, -CD161a, -CD11c, or -THY-1 antibody (Santa Cruz Biotechnology, Santa Cruz, CA, USA) and then with $10 \mu \mathrm{L} /$ $\mathrm{mL}$ rat anti-PE magnetic beads. The cell suspension was loaded onto the separation column. After the magnetic field was removed, the obtained solutions were washed with phosphatebuffered saline at $4^{\circ} \mathrm{C}$ and used as the suspension for BECs, OCs, HSCs, SECs, KCs, PCs, and DCs (Gee et al., 1991; Chalmers et al., 1998; Joplin and Kachilele, 2009). Finally, anti-ALB and G6P, CK18 and GGT1, OC2 and OV6, DES and VIM, CD14 and ET-1, LYZ and ED2, CD8 and CD56, and CD86 and CD103 antibodies were used to identify HCs, BECs, OCs, HSCs, SECs, KCs, PCs, and DCs, respectively, as previously described (Figure S1). The purity of the 8 cell types after isolation was determined (Figure S2).

\section{RNA isolation and purification}

Total RNA was isolated from hepatic cell suspensions according to the Trizol reagent manual (Invitrogen, Carlsbad, CA, USA) and then purified following the RNeasy miniprotocol (Qiagen, Hilden, Germany) (Norton, 1992). The purity of extracted total RNA was determined based on the $\mathrm{A}_{260} / \mathrm{A}_{280}$ ratio (Scott, 1995).

\section{Rat Genome 2302.0 microarray detection and data analysis}

Transcription profiles of the 8 types of regenerating hepatic cells were determined using Rat Genome 2302.0 chips according to previously described protocols for cDNA synthesis, in vitro transcription to produce biotin-labeled cRNA, hybridization of cRNA to the chips, scanning of image output files, and microarray data analysis (Kube et al., 2007; Xiao et al., 2008). The genes showing greater than 3 -fold changes in expression for at least 1 time 
point during LR with a significant difference $(0.01 \leq \mathrm{P}<0.05)$ or an extremely significant difference $(\mathrm{P} \leq 0.01)$ between $\mathrm{PH}$ and $\mathrm{OC}$, were considered to be associated with LR. Finally, these values were analyzed using GeneMath, GeneSpring (Silicon Genetics, San Carlos, CA, USA), the Microsoft Excel Software (Microsoft, Redmond, WA, USA), and Pathwaystudio 5.0 (Altschul et al., 1990; Eisen et al., 1998; Kim et al., 2008).

\section{5-Hydroxytryptamine signaling pathway gene identification}

According to the classification of physiological activity from the website Gene Ontology database (www.geneontology.org), we input the terms in the National Center for Biotechnology Information (www.ncbi.nlm.nih.gov) and Rat Genome Database (rgd.mcw.edu) to identify related genes in rat, mouse, and human. Influential genes were confirmed based on the biological pathway maps in GENMAPP (www.genmapp.org), KEGG (www.genome. $\mathrm{jp} / \mathrm{kegg} /$ pathway.html), and BIOCARTA (www.biocarta.com/genes/index.asp) (Osband and Cashon, 1990; Ogata et al., 1999; Doniger et al., 2003; Wang et al., 2007), and the 136 genes predicted to be 5-HT signaling pathway genes were reconfirmed. Sequentially, we compared the identified genes with genes in the Rat Genome 2302.0 Array and found that the chip contained 113 genes.

\section{Reliability of Rat Genome 2302.0 chip results}

Primer and probe sequences were designed using the Primer Express 2.0 software according to mRNA sequences of the 8 marker genes: $g 6 p c, g g t 1, o c 2, g f a p, c d 14, l y z, c d 56$, cd86 (marker genes of HCs, BECs, OCs, HSCs, SECs, KCs, PCs, and DCs, respectively) and the housekeeping gene $\beta$-actin (GenBank number: U07993, NM_053840, BG671896, NM_017009, NM_021744, L12458, NM_031521, NM_020081, and NM_031144) and synthesized by Shanghai GeneCore BioTechnologies Co., Ltd. (Shanghai, China) (Yoon et al., 2002). Next, target genes were subjected to amplification and polymerase chain reaction (PCR) amplification using a PRISM 7900 Sequence Detector (Applied Biosystems, Foster City, CA, USA) according to the operational guideline manual for QuantiTect SYBR Green RT-PCR Kit (Qiagen). The copies of target genes in the sample (in \%mL) were calculated according to standard curve, and the relative expression content was computed according to $\beta$-actin copy number (Fleige et al., 2006).

\section{RESULTS}

\section{Reliability of Rat Genome 2302.0 chip results}

The marker genes of the 8 liver cell types detected by reverse transcription-PCR showed that the marker gene of BEC ggt 1 and SEC $c d 14$ were up-regulated, marker genes of HC $g 6 p c$, HSC gfap, and DC $c d 86$ were down-regulated, and other cell marker genes were not expressed significantly. The gene expression profile in oligonucleotide arrays revealed no marked difference from reverse transcription-PCR for selected genes, indicating that the results of the chip were reliable (Figure 1). 

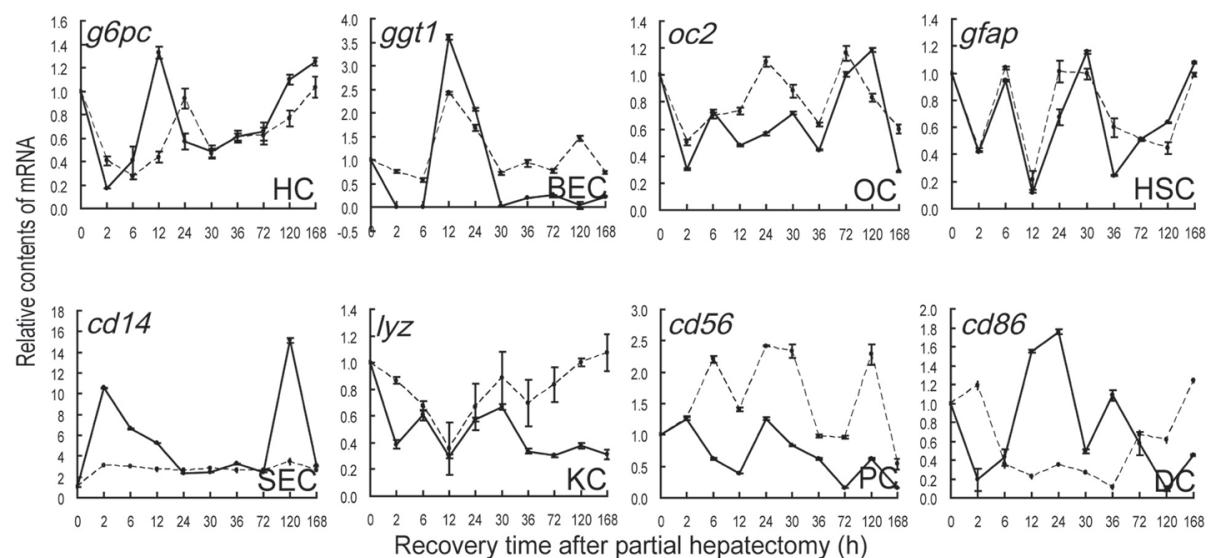

Figure 1. Dynamic changes in mRNA content of marker genes of 8 types of hepatic cells in LR. The solid line represents quantitative real-time PCR results; the broken line represents results of Rat Genome 2302.0 microarray. Hepatocytes (HCs), biliary epithelial cells (BECs), oval cells (OCs), hepatic stellate cells (HSCs), sinusoidal endothelial cells (SECs), Kupffer cells (KCs), pit cells (PCs), and dendritic cells (DCs).

\section{Expression changes in 5-HT signaling pathway genes in 8 types of liver cells and the regenerating liver}

We investigated 136 genes involved in 5-HT signaling pathways, and identified 113 of these genes in the Rat Genome 230 2.0 Array. The gene expression profiles of 8 types of liver cells and the RL tested using the chip showed that 77 genes were changed significantly. Among these, 26, 47, 8, 21, 16, 19, 22, 27, and 20 genes changed significantly in HCs, BECs, OCs, HSCs, SECs, KCs, PCs, DCs, and the RL, respectively. The numbers of up-, down-, and up/down-regulated genes in these cells were 21,5 , and $0 ; 29,10$, and $8 ; 5$, 3 , and $0 ; 8,13$, and $0 ; 11,5$, and $0 ; 8,11$, and $0 ; 19,2$, and $1 ; 16,11$, and 0 ; and 13,7 , and 0 , respectively. Of these, mapk12 and dusp6 were specifically up-regulated in HCs; in BECs, $h t r 3 a, h t r 5 a, p i k 3 c 2 b, p i k 3 r 2$, akt2, chuk, mras, and adrbkl were specifically up-regulated, pdpkl, shc1, prkarla, and creb3 were specifically down-regulated, and htr $3 b$ and gnaol were specifically up/down-regulated; in HSCs, prkce was specifically up-regulated, and camk2d and gnai 2 were specifically down-regulated; in SECs, sos 1 and grk4 were specifically up-regulated; in KCs, hras was specifically up-regulated and prkar $2 b$ was specifically down-regulated; in DCs, $h t r 7$ and rgs 12 were specifically up-regulated and nfkbie was specifically down-regulated; the expression of other genes in the 8 liver cells showed varying differences. Typically, LR is divided into 3 stages, including the initiating stage (PH after 2-6 h), progress stage ( $\mathrm{PH}$ after 6-72 h), and termination stage (PH after 72-168 h) according to the process of LR and cell physiological activities (Taub, 2004). At various time points, genes associated with LR in HCs, HSCs, SECs, KCs, and PCs began to be expressed during the initiating stage in BECs and DCs during the progress stage. Based on the observed gene expression, 5-HT signaling pathway genes were up-regulated predominantly in HCs, BECs, SECs, and the RL during the progress stage, and were down-regulated predominantly in PCs and DCs during the termination stage (Figure 2). 


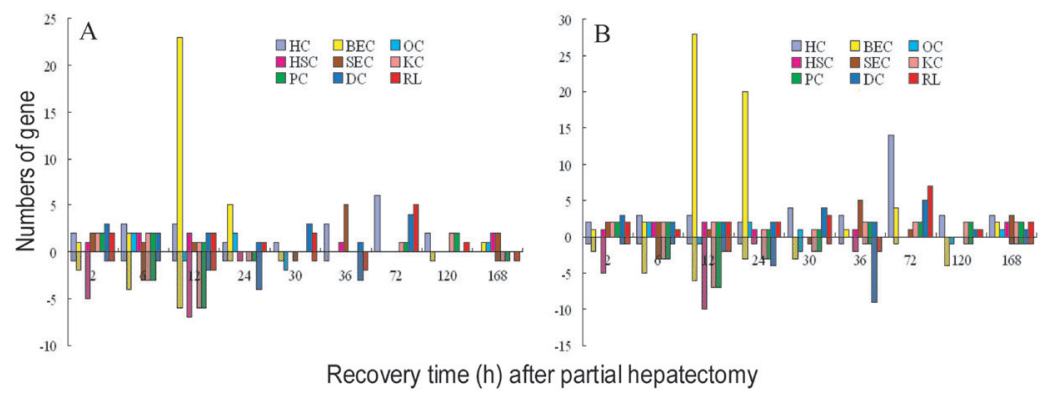

Figure 2. Expression comparison of 5-HT signaling pathway genes in 8 types of rat regenerating hepatic cells and the RL. A. Initial expression profiles. B. Total expression profiles. Empty bars, up-regulated gene; dotted bars, down-regulated genes. Hepatocytes (HCs), biliary epithelial cells (BECs), oval cells (OCs), hepatic stellate cells (HSCs), sinusoidal endothelial cells (SECs), Kupffer cells (KCs), pit cells (PCs), and dendritic cells (DCs).

\section{Gene expression differences and similarities of 6 5-HT-mediated signaling pathways in 8 types of liver cells and regenerating liver}

Studies have shown that the percentage of HCs, BECs, OCs, HSCs, SECs, KCs, PCs, and DCs accounting for the total number of liver cells were 75, 3-5, 1, 8-13, 2-3, 8-12, 0.8-1.2, and 0.3-0.6\% (Luo et al., 1995; Hisama et al., 1996; Enomoto et al., 2004; Guo et al., 2006; Sumpter et al., 2007; Quante and Wang, 2009; Lu et al., 2009). According to expression abundance of 5-HT signaling pathway genes and the proportion of cells in the liver to calculate synthetic effects of 8 types of liver cells in LR, 26 genes were changed significantly. In the RL, the gene expression trend of 10 genes such as maob and rgs 2 were the same, and that of htrlf and $h t r 2 b$ were opposite. Eight genes, including $h t r 4$ and $h t r 6$, showed significant changes in the RL, and $d d c$, $h t r 5 b$, as well as 14 other genes, showed significant expression changes in synthetic effects in the 8 types of liver cells (Table 1).

Table 1. Gene expression change comparison of the 5-HT signaling pathway at the level of tissue and cells.

\begin{tabular}{|c|c|c|c|c|c|c|c|c|c|}
\hline No. & $\begin{array}{l}\text { Gene } \\
\text { symbol }\end{array}$ & $\begin{array}{c}\text { Regenerating } \\
\text { liver }\end{array}$ & Liver cells & Comparison & No. & $\begin{array}{l}\text { Gene } \\
\text { symbol }\end{array}$ & $\begin{array}{c}\text { Regenerating } \\
\text { liver }\end{array}$ & Liver cells & Comparison \\
\hline 2 & Ddc & & $\downarrow$ & & 69 & Camk4 & & $\uparrow$ & \\
\hline 4 & Maob & $\downarrow$ & $\downarrow$ & $\sqrt{ }$ & 83 & Fyn & & $\uparrow$ & \\
\hline 5 & Aldh2 & $\downarrow$ & & & 91 & Adcy3 & $\uparrow$ & $\uparrow$ & $\sqrt{ }$ \\
\hline 10 & Htr1f & $\downarrow$ & $\uparrow$ & $x$ & 92 & Adcy 4 & & $\uparrow$ & \\
\hline 12 & $\mathrm{Htr} 2 \mathrm{~b}$ & $\downarrow$ & $\uparrow$ & $x$ & 94 & Adcy 8 & $\uparrow$ & $\uparrow$ & $\sqrt{ }$ \\
\hline 19 & Htr4 & $\uparrow$ & & & 113 & Mapk12 & & $\uparrow$ & \\
\hline 21 & $\mathrm{Htr} 5 \mathrm{~b}$ & & $\uparrow$ & & 114 & Mapk3 & $\uparrow$ & & \\
\hline 22 & Htr6 & $\uparrow$ & & & 118 & Dusp1 & & $\downarrow$ & \\
\hline 26 & Rgs2 & $\uparrow$ & $\uparrow$ & $\sqrt{ }$ & 119 & Dusp4 & $\downarrow$ & $\downarrow$ & $\sqrt{ }$ \\
\hline 27 & Rgs4 & $\uparrow$ & $\uparrow$ & $\sqrt{ }$ & 120 & Dusp6 & & $\uparrow$ & \\
\hline 31 & Ptk2b & $\uparrow$ & & & 124 & Creb1 & & $\uparrow$ & \\
\hline 34 & Pik $3 c 2 g$ & $\downarrow$ & $\downarrow$ & $\sqrt{ }$ & 126 & Creb314 & & $\uparrow$ & \\
\hline 47 & Akt3 & & $\uparrow$ & & 128 & Gnai1 & & $\uparrow$ & \\
\hline 52 & Map3k8 & $\uparrow$ & $\uparrow$ & $\sqrt{ }$ & 131 & Rgs10 & $\uparrow$ & $\uparrow$ & $\sqrt{ }$ \\
\hline 53 & $\mathrm{Bcl} 3$ & & $\uparrow$ & & 133 & Rgs14 & $\uparrow$ & & \\
\hline 54 & Nfkbia & $\uparrow$ & & & 135 & Src & $\uparrow$ & $\uparrow$ & $\sqrt{ }$ \\
\hline 62 & Plcb2 & $\downarrow$ & & & 136 & Stat3 & & $\uparrow$ & \\
\hline
\end{tabular}

*The serial number of the vertical list corresponds to the numbers in Figure 3. $\uparrow$ represents meaningful up-regulated genes; $\downarrow$ represents meaningful down-regulated genes; $\sqrt{ }$ represents the same trend in gene expression at the level of tissue and cells; $x$ represents an opposite trend in gene expression. 
Gene expression analysis of 65 -HT-mediated signaling pathways in 8 types of liver cells and the RL showed that htr4, htr6, adcy3, adcy8, and mapk3 were up-regulated in the HTR4-, HTR6-, and HTR7-mediated signaling pathways of the RL (Figure 3). htrld, htrlf, htr 5b, gnail, and scr were up-regulated in the HTR1- and HTR5-mediated signaling pathways of HCs (Figure 4), and $h t r 2 b, c a m k 2 b, c a m k 4$, mapk12, creb1, and creb3l4 were up-regulated in the HTR2-mediated signaling pathway. htrld, src, and stat 3 were up-regulated in the HTR1-mediated signaling pathway of SECs (Figure 5). htr2c, ptk2b, pik3c2g, pik3cb, akt3, $i k b k b$, and $n t k b i a$ were up-regulated in the HTR2-mediated signaling pathway of PCs (Figure 6). In addition, expression of $h$ tr $2 a$ and $h t r 2 b$ was increased at $168 \mathrm{~h}$ after $\mathrm{PH}$, but gnaq, which interacts with $h t r 2 a$ and $h t r 2 b$, was only expressed at $2 \mathrm{~h}$. htr 6 was down-regulated only at $168 \mathrm{~h}$ in KCs and 5-HT receptor genes in oval cells were not significantly expressed. 5-HT receptor genes of BECs and DCs, which regulate cell proliferation and apoptosis, were up- and down-regulated (Table 2).

A

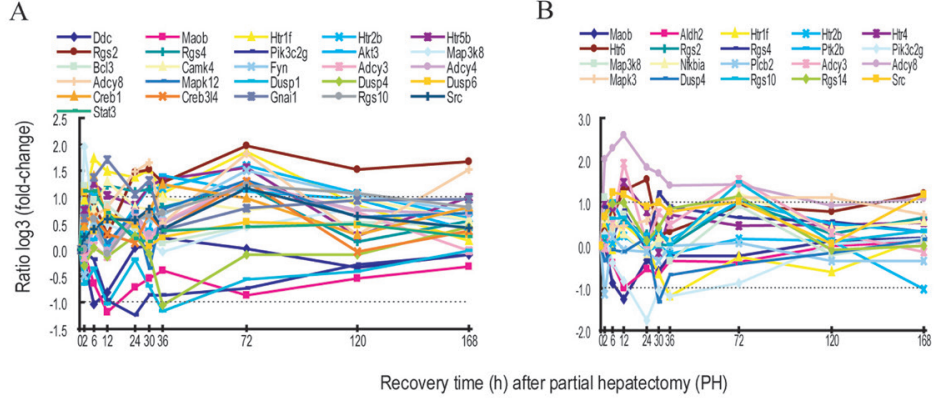

$\mathrm{C}$

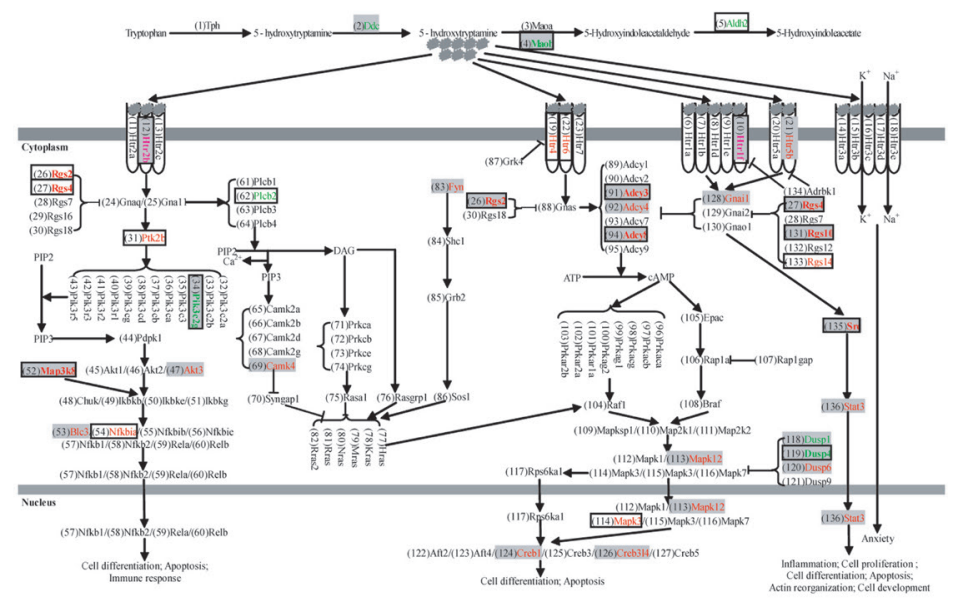

Figure 3. Gene expression changes in the 5-HT signaling pathway at the level of tissues and cells. A. mRNA temporal changes in 5-HT signaling pathway genes in the RL. B. mRNA temporal changes of 5-HT signaling pathway genes in 8 types of liver cells. C. 5-HT receptor pathways. Empty boxes indicate significantly expressed genes in the RL, Filled boxes indicate significantly expressed genes in liver cells. Red text denotes meaningful up-regulated genes; green text denotes meaningful down-regulated genes. The bold red and green text denotes the same trend in gene expression in the RL and cells, the bold pink text denotes the opposite trend of gene expression, and the black text represents meaninglessly expressed genes. 
A



B

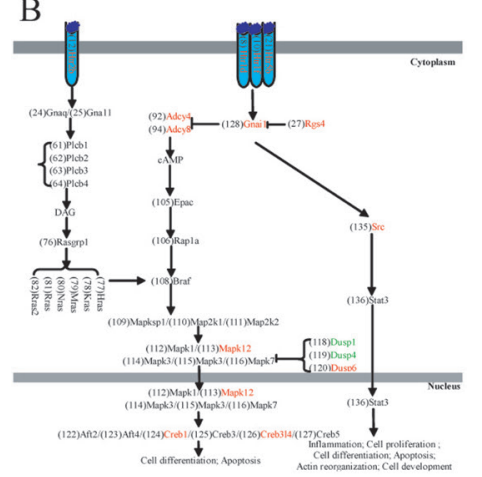

Figure 4. 5-HT signaling pathways of hepatocytes during LR. A. mRNA temporal changes in 5-HT signaling pathway genes in HC. B. 5-HT receptor pathways. Red, green, and black denote meaningful up-regulated genes, down-regulated genes, and meaninglessly expressed genes, respectively.
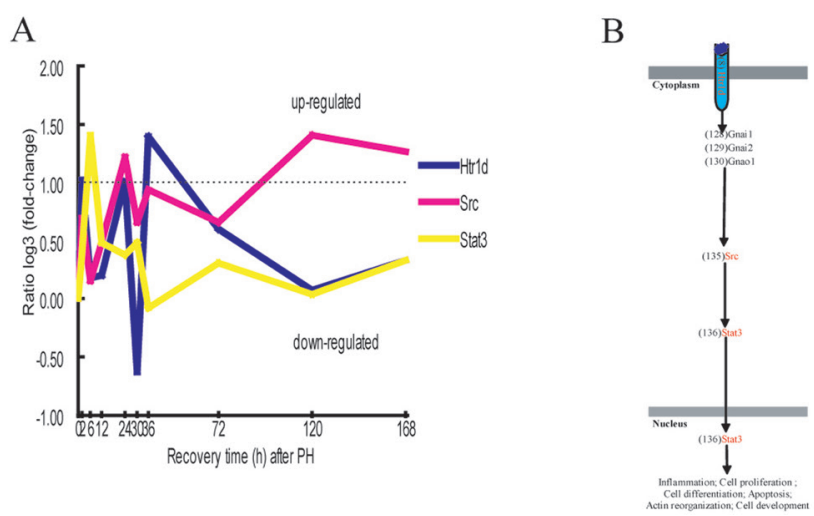

Figure 5. 5-HT signaling pathways in sinusoidal endothelial cells during LR. A. mRNA temporal changes in 5-HT signaling pathway genes in SECs. B. 5-HT receptor pathways. Red, green, and black denote meaningful upregulated genes, down-regulated genes, and meaninglessly expressed genes, respectively.
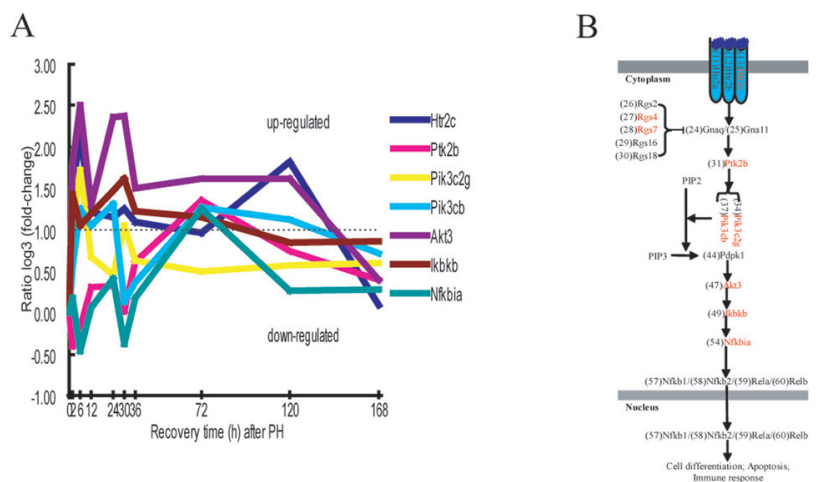

Figure 6. 5-HT signaling pathways of pit cells during LR. A. mRNA temporal changes in 5-HT signaling pathway genes in PCs. B. 5-HT receptor pathways. Red, green, and black denote meaningful up-regulated genes, downregulated genes, and meaninglessly expressed genes, respectively. 


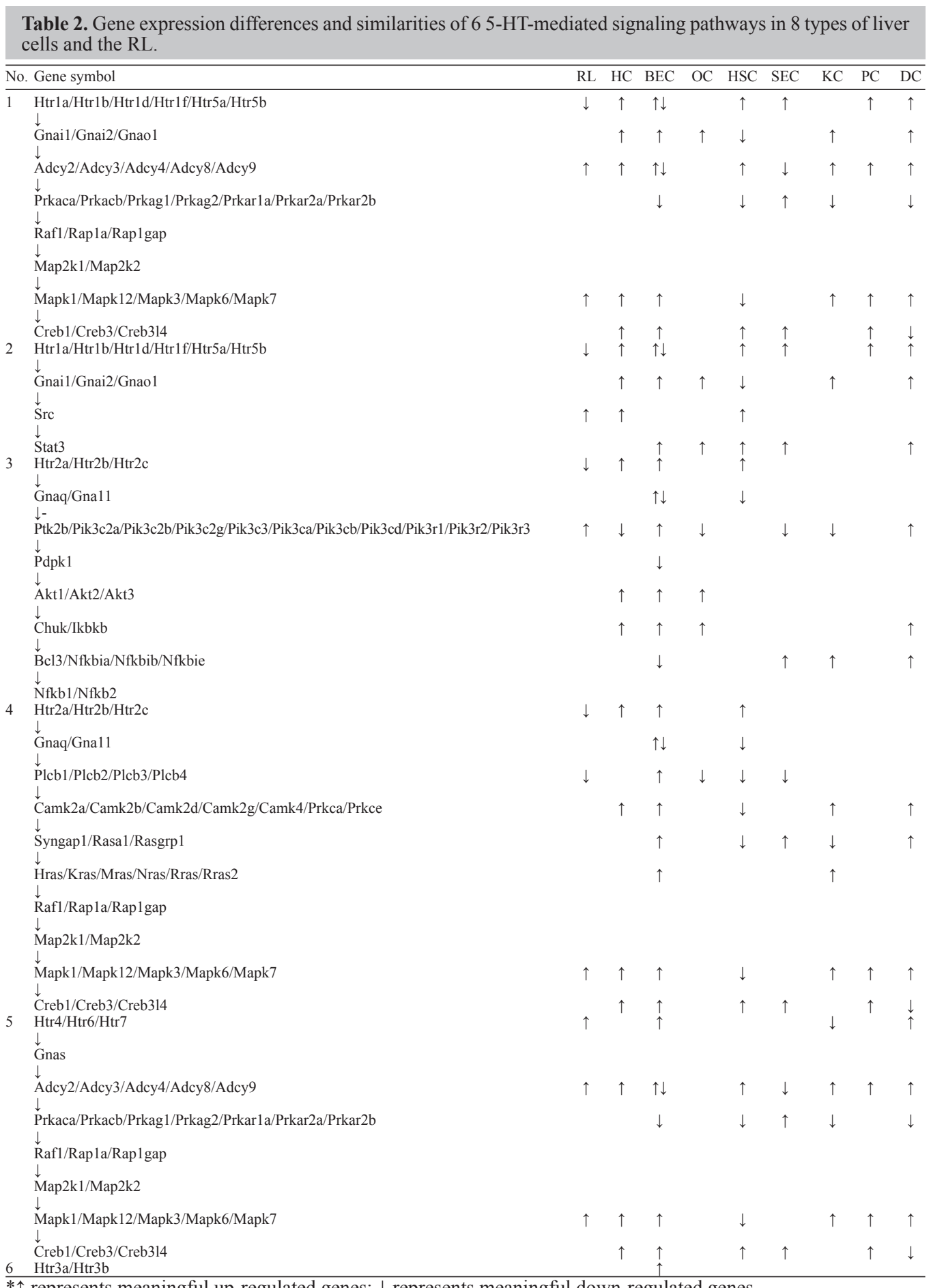

* $\uparrow$ represents meaningful up-regulated genes; $\downarrow$ represents meaningful down-regulated genes. 


\section{DISCUSSION}

Recent studies have indicated that 5-HT plays an important role in LR. A study by Papadimas et al. (2006) showed that 5-HT was synchronized to DNA synthesis after PH; Balasubramanian and Paulose (1998) showed that 5-HT promoted hepatocyte proliferation through its receptor. However, the current research of 5-HT effects on LR examined only a few genes involved in metabolism or related to receptors. To better gain insight into the role of 5-HT in LR, 8 types of high-purity and active liver cells were isolated using the combination of the Percoll density-gradient centrifugation and immunomagnetic bead methods. The Rat Genome 2302.0 Array contains $85 \%$ of the genes in the total rat genome and was used to detect gene expression profiles of 8 types of liver cells and the RL at $0,2,6,12,24,30,36,72,120$, and $168 \mathrm{~h}$ after PH. We found that 77 5-HT signaling pathway genes were involved in LR at the level of cells and tissues. Studies have shown that 5-HT regulates downstream physiological and biochemical activities mainly by binding its receptor with small $\mathrm{G}$ proteins (Hoyer et al., 1994). The liver is composed of HCs, BECs, OCs, HSCs, SECs, KCs, PCs, and DCs, among others. The comparison of the expression of 5-HT signaling pathway gene changes between 8 types of liver cells and the RL showed that $h$ tr 4 and htr 6 were up-regulated during the progress stage in RL; both genes promote cell differentiation or apoptosis through the GNAS and ERK signaling pathways. However, the synthetic effects of 8 types of liver cells showed that $h$ trlf, $h t r 2 b$, and $h t r 5 b$ were up-regulated during the termination stage of LR, in which HTR2B regulates cell differentiation and development through the GNAQ and AKT signaling pathways and HTR1F and HTR5B regulate apoptosis through GNAI by inhibiting adenylyl cyclase genes (Figure 3). These results indicate that the physiological activity of LR differs on the tissue and cellular levels, and examining single cell types is essential for LR research. Expression pattern analysis of 5-HT signaling pathway genes in HC indicated that the 5-HT receptors $h t r 2 b$ (1.44-fold), htrld (1.02-fold), htrlf (2.42-fold), and htr5b (2.08-fold), and their downstream signaling pathway genes mapk12 (1.72-fold), creb1 (1.08-fold), creb3l4 (1.57-fold), and $\operatorname{src}(1.42$-fold) were up-regulated at $72 \mathrm{~h}$ after PH (Figure 4A). Lesurtel et al. (2008) showed that mRNA expression of the 5-HT receptors $h t r 2 a$ and $h t r 2 b$ were increased after $\mathrm{PH}$, which is consistent with our results. According to the temporal dynamics of these genes, the 5-HT signaling pathway may promote cell differentiation or apoptosis through the RAS and STAT3 signaling pathways, and thus participate in regulating LR during the termination stage (Figure 4B). Expression pattern analysis of 5-HT signaling pathway genes in SEC indicated that the 5-HT type I receptor htrld (1.39-fold) and its downstream signaling pathway genes $\operatorname{src}(1.26$-fold) and stat3 (1.41-fold) were up-regulated at 36,168 , and $6 \mathrm{~h}$ after PH, respectively (Figure 5A). The results showed that the 5-HT signaling pathway may regulate sinus endothelial cell proliferation and differentiation through the STAT3 signaling pathway during LR (Figure 5B). Expression pattern analysis of 5-HT signaling pathway genes in PCs indicated that $h t r 2 c$ (2.15-fold) was up-regulated at $6 \mathrm{~h}$ after $\mathrm{PH}$, and its downstream signaling pathway genes $p t k 2 b$ (1.36-fold), pik3c2g (1.72-fold), pik3cb (1.27-fold), akt3 (2.37-fold), $i k b k b$ (1.61-fold), and ntkbia (1.25-fold) were up-regulated at 72, 6, 72, 30, 30-36, and $72 \mathrm{~h}$, respectively (Figure 6A). The results showed that the 5-HT signaling pathway can regulate PC proliferation and apoptosis through the AKT3 signaling pathway in LR (Figure 6B). In addition, $h$ tr $2 a$ and $h t r 2 b$ were up-regulated at $168 \mathrm{~h}$ after PH in HSCs, and their interaction with the small $\mathrm{G}$ protein gene gnaq was down-regulated only at $2 \mathrm{~h}$, suggesting that 5-HT may promote HSC apoptosis during the termination stage of LR through its type II receptor. The 
5-HT receptor htr6 was down-regulated only at $168 \mathrm{~h}$ after $\mathrm{PH}$ in $\mathrm{KCs}$ and no 5-HT receptor was significantly expressed in OCs, suggesting that 5-HT signaling pathways may not be important in KCs and OCs. A variety of 5-HT receptors with positive and negative roles in cell proliferation or apoptosis were up- or down-regulated significantly in BECs and DCs, indicating the presence of a more complex synergistic pathway that regulates the changes in BECs and DCs during LR. In summary, we examined the gene expression profiles of 5-HT signaling pathways at the mRNA level during LR by microarray analysis and screened 77 5-HT signaling pathway genes related to LR in the RL and 8 types of liver cells. We found that the LR regulation of the complicated 5-HT signaling pathways differed in the RL and in the 8 types of liver cells. There are 4 possible 5-HT pathways regulating cell proliferation or apoptosis of HCs, SECs, and PCs. Our results provide a theoretical basis for further studies of the role of 5-HT during LR. However, the Rat Genome 2302.0 chip mainly detects gene transcription, but not the expression and activity of proteins or enzymes, and thus may not represent the role of 5-HT on the RL and the 8 types of liver cells. We will verify the role of 5-HT on LR using a variety of qualitative and quantitative methods such as gene addition, gene silencing, Western blotting, and in situ hybridization to improve the understanding of LR.

\section{ACKNOWLEDGMENTS}

Research supported by the Natural Science Foundation of China (\#31201093), the Natural Science Foundation of Henan (\#122300413207 and \#122300410355), and Research Fund of Henan Normal University (\#11134).

\section{Supplementary material}

\section{REFERENCES}

Alpini G, Phillips JO, Vroman B and LaRusso NF (1994). Recent advances in the isolation of liver cells. Hepatology 20: 494-514.

Altschul SF, Gish W, Miller W, Myers EW, et al. (1990). Basic local alignment search tool. Mol. Biol. 215: 403-410.

Balasubramanian S and Paulose CS (1998). Induction of DNA synthesis in primary cultures of rat hepatocytes by serotonin: possible involvement of serotonin S2 receptor. Hepatology 27: 62-66.

Chalmers JJ, Zborowski M, Sun L and Moore L (1998). Flow through, immunomagnetic cell separation. Biotechnol. Prog. 14: 141-148.

Doniger SW, Salomonis N, Dahlquist KD, Vranizan K, et al. (2003). MAPPFinder: using Gene Ontology and GenMAPP to create a global gene-expression profile from microarray data. Genome Biol. 4: R7.

Duret C, Gerbal-Chaloin S, Ramos J, Fabre JM, et al. (2007). Isolation, characterization, and differentiation to hepatocytelike cells of nonparenchymal epithelial cells from adult human liver. Stem Cells 25: 1779-1790.

Eisen MB, Spellman PT, Brown PO and Botstein D (1998). Cluster analysis and display of genome-wide expression patterns. Proc. Natl. Acad. Sci. U. S. A. 95: 14863-14868.

Enomoto K, Nishikawa Y, Omori Y, Tokairin T, et al. (2004). Cell biology and pathology of liver sinusoidal endothelial cells. Med. Electron. Microsc. 37: 208-215.

Fausto N, Campbell JS and Riehle KJ (2006). Liver regeneration. Hepatology 43: S45-S53.

Fleige S, Walf V, Huch S, Prgomet C, et al. (2006). Comparison of relative mRNA quantification models and the impact of RNA integrity in quantitative real-time RT-PCR. Biotechnol. Lett. 28: 1601-1613.

Gee AP, Mansour VH and Weiler MB (1991). Effects of target antigen density on the efficacy of immunomagnetic cell separation. J. Immunol. Methods 142: 127-136.

Guo FZ, Nian H, Zhang H, Huang LY, et al. (2006). Proteomic analysis of the transition from quiescent to proliferating stages in rat liver hepatectomy model. Proteomics 6: 3075-3086.

Haber BA, Mohn KL, Diamond RH and Taub R (1993). Induction patterns of 70 genes during nine days after hepatectomy 
define the temporal course of liver regeneration. J. Clin. Invest. 91: 1319-1326.

Higgins GM and Anderson RM (1931). Experimental pathology of the liver: restoration of the liver of the white rat following partial surgical removal. Arch. Pathol. 12: 186-202.

Hisama N, Yamaguchi Y, Ishiko T, Miyanari N, et al. (1996). Kupffer cell production of cytokine-induced neutrophil chemoattractant following ischemia/reperfusion injury in rats. Hepatology 24: 1193-1198.

Hoyer D, Clarke DE, Fozard JR, Hartig PR, et al. (1994). International Union of Pharmacology classification of receptors for 5-hydroxytryptamine (serotonin). Pharmacol. Rev. 46: 157-203.

Joplin R and Kachilele S (2009). Human intrahepatic biliary epithelial cell lineages: studies in vitro. Methods Mol. Biol. 481: 193-206.

Jurima-Romet M, Huang HS, Paul CJ and Thomas BH (1991). Enalapril cytotoxicity in primary cultures of rat hepatocytes. II. Role of glutathione. Toxicol. Lett. 58: 269-277.

Jurima-Romet M, Abbott FS, Tang W, Huang HS, et al. (1996). Cytotoxicity of unsaturated metabolites of valproic acid and protection by vitamins $\mathrm{C}$ and $\mathrm{E}$ in glutathione-depleted rat hepatocytes. Toxicology 112: 69-85.

Kim HJ, Park SH, Lee TH, Nahm BH, et al. (2008). Microarray detection of food-borne pathogens using specific probes prepared by comparative genomics. Biosens. Bioelectron. 24: 238-246.

Kreamer BL, Staecker JL, Sawada N, Sattler GL, et al. (1986). Use of a low-speed, iso-density Percoll centrifugation method to increase the viability of isolated rat hepatocyte preparations. In Vitro Cell Dev. Biol. 22: 201-211.

Kube DM, Savci-Heijink CD, Lamblin AF, FnKosari, et al. (2007). Optimization of laser capture microdissection and RNA amplification for gene expression profiling of prostate cancer. BMC Mol. Biol. 8: 25.

Lesurtel M, Graf R, Aleil B, Walther DJ, et al. (2006). Platelet-derived serotonin mediates liver regeneration. Science 312: 104-107.

Lesurtel M, Soll C, Graf R and Clavien PA (2008). Role of serotonin in the hepato-gastrointestinal tract: an old molecule for new perspectives. Cell Mol. Life Sci. 65: 940-952.

Lu Q, Xie LF, Wang L, Fan JY, et al. (2009). Isolation, Purification and Identification of Hepatic Oval Cells in Rat Liver. Henan Sci. 27: 1219-1223.

Luo D, Vanderkerken K, Bouwens L, Kuppen PJ, et al. (1995). The number and distribution of hepatic natural killer cells (pit cells) in normal rat liver: an immunohistochemical study. Hepatology 21: 1690-1694.

Michalopoulos GK (2007). Liver regeneration. J. Cell Physiol. 213: 286-300.

Norton JN (1992). Total RNA isolation by a rapid centrifugation method. Am. Biotechnol. Lab. 10: 41.

Ogata H, Goto S, Sato K, Fujibuchi W, et al. (1999). KEGG: Kyoto Encyclopedia of Genes and Genomes. Nucleic Acids Res. 27: 29-34.

Osband ME and Cashon GW (1990). Biocare: biotechnology in the clinical practice of medicine. Clin. Res. 38: 5-9.

Papadimas GK, Tzirogiannis KN, Panoutsopoulos GI, Demonakou MD, et al. (2006). Effect of serotonin receptor 2 blockage on liver regeneration after partial hepatectomy in the rat liver. Liver Int. 26: 352-361.

Quante M and Wang TC (2009). Stem cells in gastroenterology and hepatology. Nat. Rev. Gastroenterol. Hepatol. 6: 724-737.

Scott RJ (1995). Isolation of whole cell (total) RNA. Methods Mol. Biol. 49: 197-202.

Sumpter TL, Abe M, Tokita D and Thomson AW (2007). Dendritic cells, the liver, and transplantation. Hepatology 46: 2021-2031.

Taub R (2004). Liver regeneration: from myth to mechanism. Nat. Rev. Mol. Cell Biol. 5: 836-847.

Vondran FW, Katenz E, Schwartlander R, Morgul MH, et al. (2008). Isolation of primary human hepatocytes after partial hepatectomy: criteria for identification of the most promising liver specimen. Artif. Organs 32: 205-213.

Wang JZ, Du Z, Payattakool R, Yu PS, et al. (2007). A new method to measure the semantic similarity of GO terms. Bioinformatics 23: 1274-1281.

Xiao P, Tang A, Yu Z, Gui Y, et al. (2008). Gene expression profile of 2058 spermatogenesis- related genes in mice. Biol. Pharm. Bull. 31: 201-206.

Yoon JR, Laible PD, Gu M, Scott HN, et al. (2002). Express primer tool for high-throughput gene cloning and expression. Biotechniques 33: 1328-1333. 